\title{
LIDAR DETECTION OF FISH SCHOOLS
}

\author{
James H. Churnside \\ NOAA Environmental Technology Laboratory \\ R/E/ET1 \\ 325 Broadway \\ Boulder, CO 80303 \\ phone: (303) 497-6744 \\ fax: (303) 797-3577 \\ email: jchurnside@etl.noaa.gov
}

\begin{abstract}
Direct measurement of fish populations in the open ocean is difficult at best. Ship-based technologies, such as trolling and acoustic sounding, are limited in coverage by the speed of the surface ship. Current airborne techniques, such as photography and direct visual observation, are not very quantitative and are limited in depth coverage to the top few meters, depending on conditions. We are developing lidar to provide an air-borne technology that will provide quantitative information about fish schools to depths of a few tens of meters. A Q-switched, frequency-doubled Nd:YAG laser provides 15 -nsec pulses of $532-\mathrm{nm}$ light. The backscattered return is collected by a telescope, detected, and digitized. The digitized signal is processed to separate fish-school returns from returns from other scatters in the water. We have made lidar observations of fish schools from a ship and from aircraft. We have measured fish reflectivity in a sea-water tank in order to convert lidar return into quantitative biomass statistics. We are currently working on algorithms for automatic detection and classification of schools.
\end{abstract}

\section{INTRODUCTION}

That fish schools can be detected by lidar has been known for some time [1]. As a short pulse of light propagates down through the ocean, it is absorbed and scattered by sea water and particles, including large particles such as fish. A fraction of the scattered light will travel back toward the source, where it can be collected and detected. As schools of fish generally have a greater optical density than the surrounding water, they will stand out from the surrounding water in the detected signal. This provides the basis for lidar detection of fish schools.

Although lidar is of interest because it can be operated from an aircraft and can therefore cover a large area of the ocean in a short time, our first experiments were conducted from a ship [2]. This allowed us to perform direct comparisons between the

This work was partially supported by a joint National Oceanic and Atmospheric Administration - Department of Defense Advanced Sensor Applications Program lidar and the echo-sounder on the ship, and also to physically sample target schools (by trawl) to verify their composition. More recently, we have operated the system from aircraft over schools of anchovy and herring. Much of the effort has been directed toward distinguishing between fish schools and other scatterers.

\section{SIGNAL PROCESSING}

In water with a uniform distribution of small scatterers, the magnitude of the lidar signal will drop exponentially with depth. The presence of fish at some depth will increase the signal returned from that depth. The result is a lidar signal that can be modeled as:

$$
S(z)=a\left[1+\frac{\beta_{f}(z)}{\beta_{w}}\right] \frac{\exp (-2 \alpha z)}{\left(n h \frac{\cos \theta_{w}}{\cos \theta_{a}}+z\right)^{2}}+b,
$$

where $S$ is the signal level, $z$ is depth, $a$ is an amplitude factor (that includes such things as laser power, telescope diameter, and receiver gain), $\beta_{\mathrm{f}}$ is the backscattering coefficient of the fish at a given depth, $\beta_{w}$ is the backscattering coefficient of the water and small particulates, $\alpha$ is an attenuation coefficient, $n$ is the index of refraction of sea water, $h$ is the height of the source above the surface, $\theta_{w}$ is the angle from nadir of the laser beam in the water, $\theta_{\mathrm{a}}$ is the angle in air, and $b$ is an offset caused by background light and any electronics offsets.

A typical lidar return, including fish, is presented in Fig. 1. The solid line is the actual return, and the dashed line is the equation given above with $\beta_{\mathrm{f}}$ assumed to be zero. The large difference between the measured curve and the equation at about $15 \mathrm{~m}$ depth is due to the return from a school of fish. Note that the return is very small at depths less than $5 \mathrm{~m}$. Very near the surface, the lidar return is contaminated by specular reflections from the surface. For the measurements from the ship, including the data in the figure, this problem was overcome by switching the detector on at a time corresponding to a depth of $5 \mathrm{~m}$. This caused two artifacts in the data, one at 


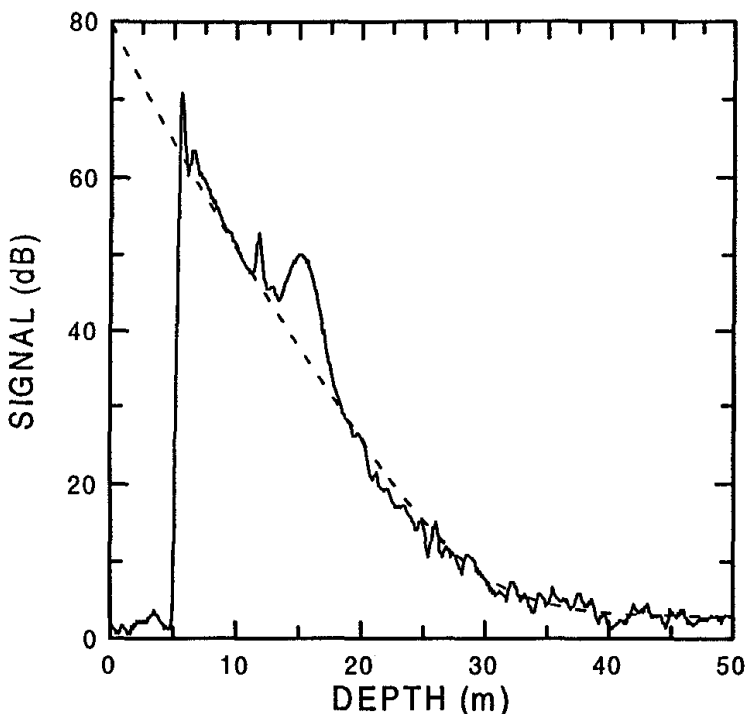

Fig. 1. Typical return from a lidar pulse taken from the ship.

$5 \mathrm{~m}$ and another at about $12 \mathrm{~m}$. These two peaks were very constant and were easily ignored. For the airborne data, the surface return was recorded, but the top $5 \mathrm{~m}$ were not considered during signal processing.

A grey-scale image similar to that from an echo sounder can be obtained by subtracting the equation result with $\beta_{\mathrm{f}}=0$ from the actual signal. This was done to visualize fish schools.

Each return can also be fit to the equation with some assumption about the depth distribution of $\beta_{\mathrm{f}}$. We used a Gaussian shape, which seemed to work fairly well under most conditions. The advantage of this processing step is that it allows the process of recognizing fish schools in the data to be automated. This has not been done for the data from the ship, but for the data collected from aircraft, we applied the criteria that a school of fish should have a contrast $\left(\beta_{\mathrm{f}} / \beta_{\mathrm{w}}\right)$ greater than 1 for 5 consecutive shots. This seemed to work fairly well, but more work needs to be done to refine the process.

\section{RESULTS}

The ship-based data were taken from the R/V David Starr Jordan in September, 1995 in the Southern California Bight. Before the cruise, calibration measurements were made on about 50 sardines in a 10-m-deep sea water tank. The equivalent diffuse reflection was measured to be $10 \%$ for the return co-polarized with the transmitted laser and about $3 \%$ for the receiver cross-polarized with respect to the transmitter. The small-particle return was estimated for each lidar pulse and subtracted from the actual return signal. These signals were averaged for 1-hour periods, and the calibration factor based on the measured reflectivity was applied. For most of the data, a cross-polarized return was used. This produced a lower signal, but better contrast between fish and small-particulate returns.

A typical result is shown in Fig. 2. This figure presents the habitat density (in $\mathrm{kg} \mathrm{m}^{-3}$ ) for one hourly period. Note the presence of several distinct layers. An estimate of the total biomass in the survey area can be made by integrating over depth and multiplying by the survey area.

In April 1997, the lidar was installed on a Beech King Air owned and operated by the California State Department of Fish and Game. For these data, the presence of fish in data files was identified using the criteria of contrast greater than unity for 5 consecutive lidar pulses. Other than that, the Gaussian fit to the fish return was not used.

Fig. 3 presents the grey-scale image of a school of anchovies taken from the plane. It represents 30 seconds of data, which corresponds to about $2.1 \mathrm{~km}$. The data from the top 5 meters are not shown because they are contaminated by the surface return. A plankton layer extended from the 5-m level down to about 30 meters. This extended for many miles of the flight path, and is shown in the right half of the figure. The darker regions in the left portion of the figure are several schools of anchovies. The largest of these appears to be about $80 \mathrm{~m}$ in diameter. The entire group of schools extends over about 900 $m$. The identification of these schools as anchovies was provided by the R/V David Starr Jordan.

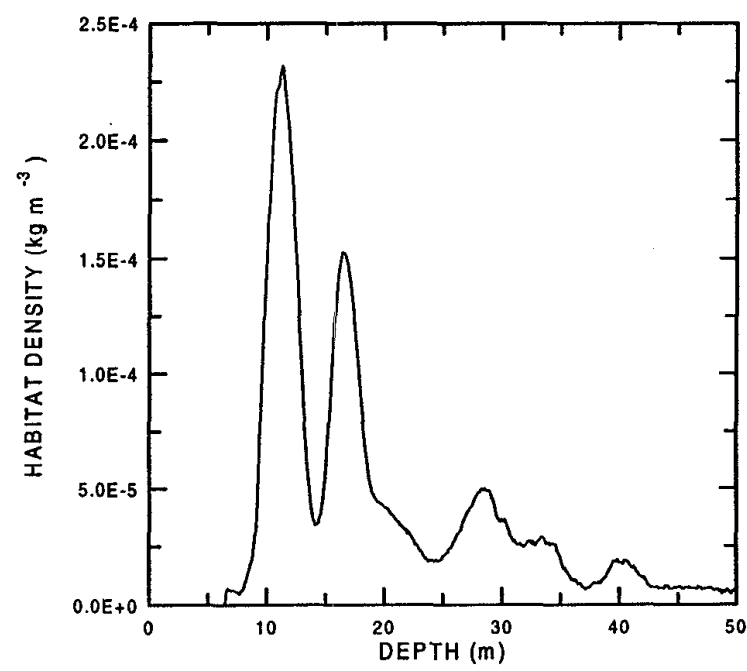

Fig. 2. Fish density for the habitat surveyed from 0800 to 0900 PDT, 24 September, 1995. 


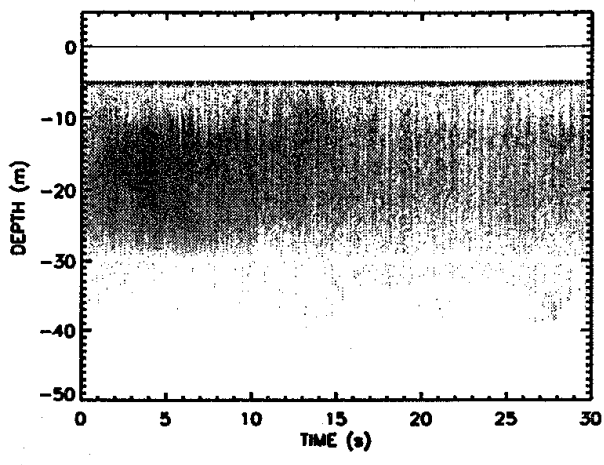

Fig. 3. Lidar image of fish schools.

\section{CONCLUUSIONS}

Schools of fish in the top few tens of meters of the ocean can be profiled with an airborne lidar. The spatial distribution over large areas of the ocean can be quickly sampled using this technique. The spatial distribution of profiles can be used to estimate the total biomass of fish using the appropriate calibration factors.
Several areas of further research remain. The temporal response of the system can be increased so that schools closer to the surface can be detected. The dynamic range can be increased to extend the depth penetration. The algorithms used to estimate the small-particulate scattering can be improved, especially in the case of non-uniform distributions of scatterers. The algorithms used to detect the presence of fish can be improved, especially in the way in which data from multiple lidar pulses are used.

\section{ACKNOWLEDGMENTS}

I would like to express my appreciation to Drs. John Hunter and Paul Smith of NOAA's Southwest Fisheries Science Center, the officers and crew of the NOAA R/V David Starr Jordan, and the pilots of the California State Department of Fish and Game.

\section{REFERENCES}

[1] J. L. Squire, Jr. and H. Krumboltz, "Profiling pelagic fish schools using airborne optical lasers and other remote sensing techniques," Mar. Technol. Soc. J. 15, 27-31, 1981.

[2 ] J. H. Churnside, J. J. Wilson, and V. V. Tatarskii, "Lidar profiles of fish schools," Appl. Opt. 36, 6011-6020, 1997. 